\title{
Stage IIIA Cervical Cancer FIGO 2018
}

National Cancer Institute

\section{Source}

National Cancer Institute. Stage IIIA Cervical Cancer FIGO 2018. NCI Thesaurus. Code C162240.

Cervical carcinoma that involves the lower third of the vagina, with no extension to the pelvic wall. (FIGO 2018) 